\title{
Intracellular Mycoplasma genitalium infection of human vaginal and cervical epithelial cells elicits distinct patterns of inflammatory cytokine secretion and provides a possible survival niche against macrophage-mediated killing
} Chris L McGowin ${ }^{1}$, Vsevolod L Popov ${ }^{1}$ and Richard B Pyles*1,2

Address: ${ }^{1}$ Department of Pathology, University of Texas Medical Branch, 301 University Blvd., Galveston, TX, 77555-0609, USA and ${ }^{2}$ Department of Pediatrics, Sealy Center for Vaccine Development, University of Texas Medical Branch, 301 University Blvd., Galveston, TX, 77555-0436, USA

Email: Chris L McGowin - clmcgowi@utmb.edu; Vsevolod L Popov - vpopov@utmb.edu; Richard B Pyles* - rbpyles@utmb.edu

* Corresponding author

Published: 14 July 2009

BMC Microbiology 2009, 9:139 doi:10.1/86/147/-2180-9-139
Received: 27 January 2009

Accepted: 14 July 2009

This article is available from: http://www.biomedcentral.com/147/-2180/9/139

(c) 2009 McGowin et al; licensee BioMed Central Ltd.

This is an Open Access article distributed under the terms of the Creative Commons Attribution License (http://creativecommons.org/licenses/by/2.0), which permits unrestricted use, distribution, and reproduction in any medium, provided the original work is properly cited.

\begin{abstract}
Background: Mycoplasma genitalium is an emerging sexually transmitted pathogen that has been associated with significant reproductive tract inflammatory syndromes in women. In addition, the strong association between severity of $M$. genitalium infection and Human Immunodeficiency Virus type I (HIVI) shedding from the cervix suggests that innate responses to $M$. genitalium may influence pathogenesis of other sexually transmitted infections. Epithelial cells (ECs) of the reproductive mucosa are the first cells contacted by sexually transmitted pathogens. Therefore, we first characterized the dynamics of intracellular and extracellular localization and resultant innate immune responses from human vaginal, ecto- and endocervical ECs to M. genitalium type strain G37 and a low-pass contemporary isolate, M2300.

Results: Both M. genitalium strains rapidly attached to vaginal and cervical ECs by $2 \mathrm{~h}$ post-infection (PI). By $3 \mathrm{~h} \mathrm{PI}, M$. genitalium organisms also were found in intracellular membrane-bound vacuoles of which approximately $60 \%$ were adjacent to the nucleus. Egress of $M$. genitalium from infected ECs into the culture supernatant was observed but, after invasion, viable intracellular titers were significantly higher than extracellular titers at 24 and $48 \mathrm{~h} \mathrm{Pl}$. All of the tested cell types responded by secreting significant levels of pro-inflammatory cytokines and chemokines in a pattern consistent with recruitment and stimulation of monocytes and macrophages. Based on the elaborated cytokines, we next investigated the cellular interaction of $M$. genitalium with human monocyte-derived macrophages and characterized the resultant cytokine responses. Macrophages rapidly phagocytosed $M$. genitalium resulting in a loss of bacterial viability and a potent pro-inflammatory response that included significant secretion of IL-6 and other cytokines associated with enhanced HIV-I replication. The macrophage-stimulating capacity of $M$. genitalium was independent of bacterial viability but was sensitive to heat denaturation and proteinase-K digestion suggesting that $M$. genitalium protein components are the predominant mediators of inflammation.

Conclusion: Collectively, the data indicated that human genital ECs were susceptible and immunologically responsive to $M$. genitalium infection that likely induced cellular immune responses. Although macrophage phagocytosis was an effective method for $M$. genitalium killing, intracellular localization within vaginal and cervical ECs may provide $M$. genitalium a survival niche and protection from cellular immune responses thereby facilitating the establishment and maintenance of reproductive tract infection.
\end{abstract}




\section{Background}

Mycoplasma genitalium is now recognized as a sexually transmitted pathogen $[1,2]$. In healthy young men and women, the prevalence of $M$. genitalium infection is approximately $1 \%$ and is between those of gonococcal $(0.4 \%)$ and Chlamydia trachomatis (4.2\%) infections [2]. In men, M. genitalium is a recognized and important cause of non-gonococcal urethritis [3]. Reproductive tract disease syndromes associated with M. genitalium infection in women include pelvic inflammatory disease [4] and cervicitis [5-9]. Limited serologic studies and detection of $M$. genitalium DNA in cervical, endometrial and/or Fallopian tube specimens from women with salpingitis [10] have suggested that $M$. genitalium could also be a cause of tubal factor infertility $[11,12]$ independent of Chlamydia trachomatis. Importantly, the burden of M. genitalium at the cervical mucosa is positively correlated with Human Immunodeficiency Virus type 1 (HIV-1) shedding [13] but the cell types involved and the mechanisms of these associations remain unclear. Select pro-inflammatory cytokines, including IL-6, have been associated with increased HIV-1 titers [14] and up-regulate HIV-1 replication [15]. These findings indicate that M. genitalium infection may enhance acquisition or dissemination of other sexually transmitted infections and provide strong rationale for investigation into the host innate immune response.

The mucosal surfaces of the female reproductive tract provide a physical barrier against invading pathogens. Importantly, these surfaces are adapted to constant antigenic stimulation from the normal polymicrobial flora but are concomitantly charged with recognition and response to pathogen exposure. Following sexual transmission, $M$. genitalium and other pathogens make initial contact with epithelial cells (ECs) that play an important role in early activation of the innate response. ECs of the vagina and cervix express robust levels of Toll-like receptor (TLR) 2, 3, 5, 6 and CD14 with low levels of TLR1, 4 and 7-9 [16]. Furthermore, both vaginal and cervical ECs recognize bacterial ligands via TLR2/6 such as the macrophage-activating lipopeptide of Mycoplasma fermentans [17]. Although macrophages are not always resident in the vaginal lumen, they are distributed throughout the epithelial and sub-epithelial mucosa of the vagina and cervix and make up a significant proportion of the total immune cell population of the reproductive tract [18]. Generally, macrophages recognize, phagocytose and destroy pathogenic bacteria [19] and studies are needed to address directly the interaction of $M$. genitalium with human macrophages. Specifically, it currently is unclear whether infection of reproductive tract ECs elicits chemokine secretion for recruitment of phagocytic cells to infected tissues resulting in inflammation.
Lipoprotein-enriched detergent phase preparations from M. genitalium strain $\mathrm{G} 37$ have been reported to activate inflammatory cytokine secretion from a transformed monocytic cell line $[20,21]$ but these fractions have yet to be tested using human genital ECs or cell types more relevant to genital transmission. Recently, our group has shown that human reproductive tract ECs are highly responsive to TLR2/6-activating regions of the MG309encoded protein resulting in inflammatory cytokine secretion [22]. To further explore the responses of human genital ECs, we have established that $M$. genitalium inoculation of human vaginal, ecto- and endocervical ECs results in both extra- and intracellular infection that elicits tissue-specific cytokine secretion. The elaborated cytokines were consistent with recruitment of macrophages to the reproductive mucosa. In addition, subsequent testing showed that human monocyte-derived macrophages (MDM) rapidly phagocytosed and killed $M$. genitalium resulting in a robust secretion of pro-inflammatory cytokines. These data provide the first characterization of the human innate immune response to viable $M$. genitalium from relevant cell types of the female reproductive tract and provide insight into the dynamic interaction with the reproductive mucosa.

\section{Methods \\ Human cell culture}

Immortalized human ECs derived from vaginal $(n=3$ donors; V19I, V12I, V11I), ectocervical and endocervical tissues were maintained as described previously [16]. Keratinocyte serum-free medium (KSFM; Invitrogen, Carlsbad, CA) supplemented with bovine pituitary extract (50 mg/L), recombinant epidermal growth factor (5 ug/ $\mathrm{L}), \mathrm{CaCl}_{2}(44.1 \mathrm{mg} / \mathrm{L})$, penicillin-G $(100 \mathrm{U} / \mathrm{mL})$ and streptomycin sulfate $(100 \mathrm{ug} / \mathrm{mL})$ was used for culture of ectocervical and endocervical ECs at $37^{\circ} \mathrm{C}$ in a $5 \% \mathrm{CO}_{2}$ humidified incubator [23]. Vaginal ECs were maintained in a 1:1 mixture of KSFM and VEC-100 media (MatTek, Ashland, MA). ME-180 (ATCC HTB-33) cervical carcinoma cells were maintained in RPMI 1640 (MediaTech, Herndon, VA) medium supplemented with $0.1 \mathrm{mM}$ nonessential amino acids (Sigma-Aldrich, St. Louis, MO), 2 mM L-glutamine, penicillin-G $(100 \mathrm{U} / \mathrm{mL})$, streptomycin sulfate $(100 \mathrm{ug} / \mathrm{mL}$ ) and $10 \%$ fetal bovine serum (FBS; Invitrogen). Cells were verified to be free of any contaminating mycoplasmas by PCR (Stratagene, Cedar Creek, Texas).

\section{Propagation of M. genitalium strains G37 and M2300}

Mycoplasma genitalium type strain G37 (ATCC 33530) or the more contemporary, lower passage Danish M2300 strain was propagated in Friis FB medium [24]. Briefly, $M$. genitalium stocks (stored at $-80^{\circ} \mathrm{C}$ ) were inoculated aseptically into tightly sealed tissue culture flasks containing 
freshly prepared Friis FB medium and incubated at $37^{\circ} \mathrm{C}$ for $5-8 \mathrm{~d}$. Growth was monitored by the formation of adherent microcolonies and a $\mathrm{pH}$-mediated color change of the medium. M. genitalium was harvested from culture flasks by pouring off the spent medium, extensively washing adherent mycoplasmas with 5 volumes of approximately $5 \mathrm{~mL}$ each of sterile PBS and then scraping adherent microcolonies into fresh PBS. M. genitalium viability was quantified in 96-well plates by serial 10 -fold dilution of each sample into fresh Friis FB medium. The last dilution to show a change in color and formation of microcolonies was used to calculate the approximate number of viable organisms in the original sample. UVinactivation $(254 \mathrm{~nm})$ of $M$. genitalium was performed using a Stratalinker 2400 (Stratagene, La Jolla, CA) to a total energy of 720,000 microjoules $/ \mathrm{cm}^{2}$. Heat denaturation of M. genitalium was accomplished by incubating logphase cultures at $80^{\circ} \mathrm{C}$ for $15 \mathrm{~min}$ and then rapid cooling on ice. Loss of viability was verified by an absence of growth in Friis FB medium after $14 \mathrm{~d}$ incubation at $37^{\circ} \mathrm{C}$.

\section{Isolation of human monocyte-derived macrophages}

Human macrophages were generated as described previously [25] from peripheral blood mononuclear cells (PBMC) collected from healthy volunteers with University of Texas Medical Branch Institutional Review Board approval. Briefly, PBMCs were isolated using HypaqueFicoll (Amersham Biosciences, Piscataway, NJ) densitygradient separation. Selection was performed using the magnetic column separation system (StemCell Technologies, Vancouver, Canada). Purified monocytes were differentiated into macrophages by culturing in RPMI 1640 medium supplemented with $10 \%$ FBS, L-glutamine, HEPES, sodium pyruvate and GM- CSF (100 ng/mL). Following $7 \mathrm{~d}$ of differentiation, monocyte-derived macrophages (MDM) were removed from the culture plastic using a non-enzymatic cell dissociation solution (cat \# C1544, Sigma-Aldrich) and then resuspended in fresh RPMI 1640 medium. Macrophage differentiation was verified by flow cytometric confirmation of CD11b, CD80 and CD86 expression showing typical purities of $>95 \%$ (data not shown). Macrophages were differentiated from PBMCs collected from 3 different blood donors and used in 3 independent experiments.

\section{Electron Microscopy}

\section{Transmission electron microscopy}

Adherent monolayers of M. genitalium-inoculated (G37 or M2300; MOI 100) or non-inoculated genital ECs or human MDM (MOI 100) were fixed at indicated times from $2-48 \mathrm{~h}$ post-infection (PI) in a mixture of $2.5 \%$ formaldehyde and $0.1 \%$ glutaraldehyde in $0.05 \mathrm{M}$ cacodylate buffer (pH 7.2) containing $0.03 \%$ trinitrophenol and $0.03 \% \mathrm{CaCl}_{2}$. Cells were scraped, centrifuged briefly at $1,000 \times \mathrm{g}$, washed in $0.1 \mathrm{M}$ cacodylate buffer ( $\mathrm{pH} 7.2)$ and then post-fixed in $1 \% \mathrm{OsO}_{4}$ in the same buffer. Each sam- ple was stained en bloc with $1 \%$ uranyl acetate in $0.1 \mathrm{M}$ maleate buffer, dehydrated in ethanol and embedded in Poly/Bed 812 epoxy resin (Polysciences, Warrington, PA). Ultrathin sections were cut using the Ultracut $S$ ultramicrotome (Reichert-Leica). Sections were stained sequentially in $2 \%$ aqueous uranyl acetate and lead citrate and then examined in a Philips 201 or CM 100 electron microscope at $60 \mathrm{kV}$.

\section{Scanning electron microscopy}

M. genitalium-infected and non-infected control cells were fixed as described above for transmission electron microscopy (TEM) for at least $1 \mathrm{~h}$ at room temperature, postfixed in $1 \%$ OsO4 in $0.1 \mathrm{M}$ cacodylate buffer, dehydrated in ethanol, treated with hexamethyldisalazane and then air dried. Next, the coverslips were mounted on the specimen stubs and sputter coated with iridium for 40 sec in an Emitech K575X turbo sputter coater (Emitech, Houston, TX). Samples were examined in a Hitachi S4700 field emission scanning electron microscope (Hitachi High Technologies America, Electron Microscope Division, Pleasanton, CA) at $2 \mathrm{kV}$.

\section{Quantification of M. genitalium uptake by cervical epithelial cells}

Uptake of $M$. genitalium by reproductive tract ECs was assessed using the gentamicin invasion assay [26]. The sensitivity of M. genitalium strains G37 and M2300 to gentamicin was established first by inoculation of log-phase organisms into Friis FB medium with gentamicin concentrations ranging from $100-400 \mathrm{ug} / \mathrm{mL}$. No M. genitalium growth was observed at 200 or $400 \mathrm{ug} / \mathrm{mL}$ therefore a working concentration of $200 \mathrm{ug} / \mathrm{mL}$ was employed in subsequent studies to minimize EC uptake of gentamicin and subsequent killing of intracellular M. genitalium. Confirmatory studies were completed subsequently using 400 $\mathrm{ug} / \mathrm{mL}$ gentamicin. As a representative genital EC type, ME-180 cells were seeded into 96-well plates $1 \mathrm{~d}$ prior to infection at a density of $1 \times 10^{5}$ cells/well. Log-phase $M$. genitalium was inoculated onto ME-180 cells (MOI of 100 ) in triplicate. Following $3 \mathrm{~h}$ incubation, when M. genitalium appeared to be attached to and invading genital ECs (see Figure 1), the inoculum was removed and replaced with fresh medium containing gentamicin. At 15 min, 24 and $48 \mathrm{~h}$ following removal of the inoculum, culture supernatants were removed and the infected cells were washed $3 \times$ with sterile PBS. Cells then were removed from the well by scraping into Friis FB medium followed by plating serial 10-fold dilutions prepared in Friis FB medium into a 96-well plate. Outgrowth of M. genitalium from infected ME-180 cells was observed for $14 \mathrm{~d}$. The load of viable $M$. genitalium from each sample was calculated by titration as described above.

The gentamicin invasion assay also was utilized to investigate whether intracellular M. genitalium were able to 




\section{Figure I}

Cultivation of $M$. genitalium and ultrastructural analysis of attachment to vaginal epithelial cells. M. genitalium G37 or M2300 were grown to log-phase in Friis FB medium. (A) Light micrograph of attached G37 microcolonies grown in culture flasks containing Friis FB medium taken using Variable Relief Contrast (VAREL). (B) TEM micrograph of a single G37 microcolony after 3d growth in Friis FB medium showing highly variable size and morphology. (C) Within M. genitalium G37 microcolonies, an elongated tip-like structure (arrow) was observed. (D) TEM micrograph M. genitalium strain M2300 showing similar variable morphology compared to G37 and formation of an electron-dense tip structure. Log-phase $M$. genitalium were harvested from Friis medium and then inoculated onto vaginal EC monolayers for ultrastructural analysis of attachment. (E) SEM micrograph of $M$. genitalium G37 attached to vaginal ECs (2 h PI). (F) TEM micrograph of $M$. genitalium G37 attached to vaginal ECs collected $3 \mathrm{~h} \mathrm{PI}$. An electron dense core structure presumably involved in attachment and invasion of vaginal ECs is highlighted by the oval. Similar electron dense cores were observed in some tip structures and can be seen in panel $\mathrm{C}$.

escape from the infected ECs. For these experiments, ME180 cervical ECs were infected with M. genitalium and then extracellular organisms were killed with gentamicin $(2 \mathrm{~h})$ after $3 \mathrm{~h}$ of infection as described above. Infected ECs then were rinsed $3 \times$ with PBS and overlaid with fresh medium that did not contain gentamicin. At $15 \mathrm{~min}, 24$ and $48 \mathrm{~h}$ following removal of the gentamicin, culture supernatants were collected for quantification of M. genitalium that had egressed from the infected cells. Quantifi- cation was performed in triplicate experiments using the CCU assay as described above.

\section{Stimulation of genital epithelial cells or primary monocyte-derived macrophages}

Human vaginal, ectocervical or endocervical ECs were seeded into 96-well plates at a density of $1 \times 10^{5}$ cells/well. Primary human MDM were seeded into 96-well plates at $5 \times 10^{4}$ /well. Following overnight incubation at $37^{\circ} \mathrm{C}$, 
culture supernatants were removed and replaced with fresh medium to remove any constitutively secreted cytokines. Log-phase M. genitalium G37 or M2300 was harvested as described above, re-suspended in fresh PBS and then inoculated onto each cell type (MOI of 10). Controls for innate immune stimulation included the $M$. salivarium-derived TLR2/ 6 agonist, FSL-1 ( $0.1 \mathrm{ug} /$ well) or an equal volume of the PBS vehicle added to triplicate wells and processed in parallel. Secreted cytokines were quantified from culture supernatants 6 or 48 h PI via a cytometric bead array (CBA) assay using the human 27 Plex panel of cytokine targets (Bio-Rad Laboratories, Hercules, CA). For testing of $M$. genitalium viability following macrophage exposure, infected macrophages were inoculated into Friis FB medium $30 \mathrm{~min}, 2,6$ or $12 \mathrm{~h}$ PI and observed for M. genitalium outgrowth indicated by a $\mathrm{pH}-$ mediated color change and adherent microcolony formation.

\section{Statistical Analyses}

The Student's t test was used to calculate significant differences in intra- and extracellular M. genitalium titers and when comparing secretion of individual cytokines from a single cell type to basal (PBS-treated) levels. The one-way ANOVA followed by Dunnett's post-test (Prism v. 4.0, GraphPad, San Diego, CA) was used to calculate significant differences in cytokine secretion levels when more than 2 conditions were compared. Significance was indicated when $\mathrm{p}<0.05$.

\section{Results}

\section{M. genitalium ultrastructure, attachment and invasion of human genital epithelial cells}

M. genitalium strain G37 or M2300 grown to log phase in Friis medium resulted in adherent microcolony formation and were characterized by a radial gradient of colony diameter (Figure 1A). Within each microcolony, M. genitalium organisms were densely packed and highly pleomorphic (Figure 1B). Several organisms were observed that showed a tip-like structure (noted with arrows) for both the Danish M2300 strain (Figure 1C) and G37 (Figure 1D).

M. genitalium has been shown previously to occupy intracellular spaces in cultured cells of non-reproductive origin [27-29] and cells obtained clinically from vaginal swabs of M. genitalium-positive women [30]. However, the invasion dynamics for genital cell types are not well understood. Inoculation of genital ECs with $M$. genitalium strains G37 or M2300 (MOI 100 for electron microscopy) resulted in attachment to vaginal (V19I; Fig 1E) and cervical (ME-180; data not shown) ECs by 2 h PI. Attachment of M. genitalium G37 and M2300 to reproductive tract ECs was consistently characterized by a polarized electrondense core, within the M. genitalium organism [31], seen adjacent to the host cell membrane (core indicated in Figure $1 \mathrm{~F}$ ). This dense core was evident within some tip structures as shown for M2300 (Figure 1C). After $3 \mathrm{~h}$ infection, M. genitalium G37 were attached to the host cells (Figure 2; starred arrows) and also observed in intracellular vacuoles distributed throughout the cellular cytosol (Figure 2; arrows). In approximately $60 \%$ of examined cells, intracellular vacuoles were directly adjacent to the nucleus $(\mathrm{N}$; Figure 2). Similar findings were observed 6-48 h PI (data not shown) for both the G37 and M2300 strain. At these later time points, extracellular $M$. genitalium also were observed but were often in aggregates and showed no evidence of attachment or invasion of host cells. Morphologically, the intracellular and extracellular mycoplasmas were highly pleomorphic and appeared to have normal ultrastructure indicated by a dense content of ribosomes and few degraded bacterial membranes. A previously

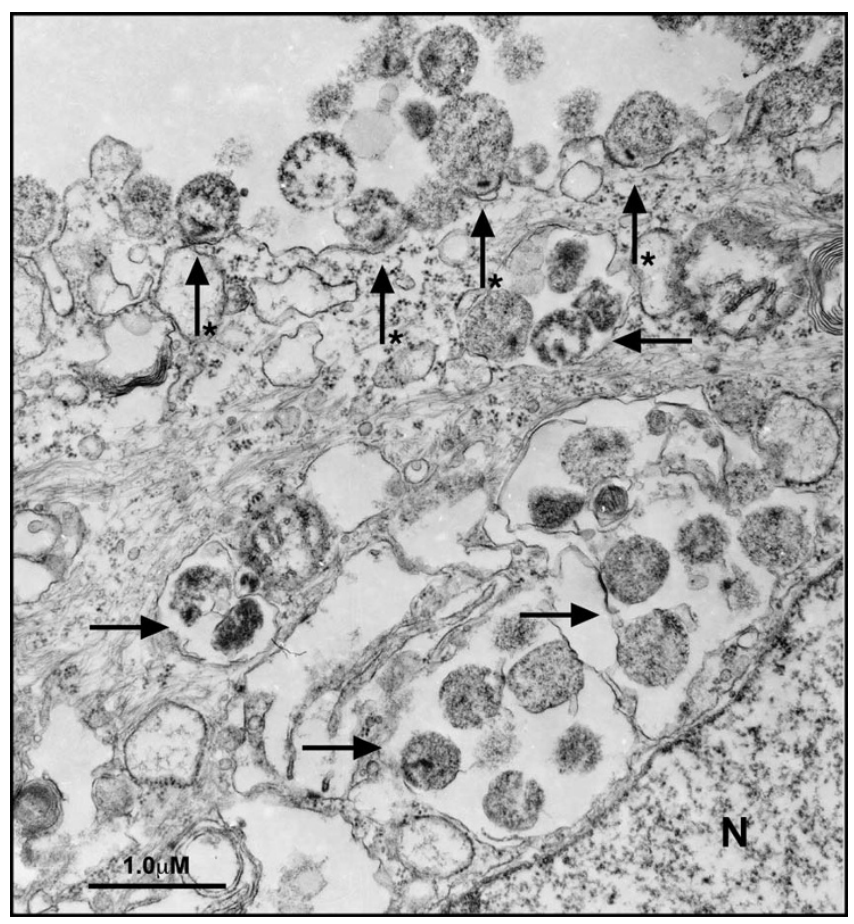

Figure 2

Attachment and invasion of vaginal epithelial cells by M. genitalium. M. genitalium G37 or M2300 were harvested from log-phase cultures in Friis FB medium and then inoculated onto vaginal ECs. After $3 \mathrm{~h}$ of infection, cells were fixed and processed for TEM imaging. Many M. genitalium organisms were attached to the host cell surface associated with a polarized electron-dense core structure (starred arrow). In addition, $M$. genitalium organisms were localized to intracellular vacuoles (arrows) distributed throughout the cellular cytosol. Approximately $60 \%$ of observed vaginal ECs showed intracellular vacuoles directly adjacent to the nucleus (denoted as N). Similar findings were observed in cervical ECs and for the Danish M2300 strain. 
described tip structure [27] was observed readily on $M$. genitalium grown in Friis FB medium (Figure 1C and 1D) but an elongated tip structure was not always visible on mycoplasmas attached to host cells in each stained section. No similar organisms or structures were observed in non-infected cells processed in parallel.

We next quantified M. genitalium G37 and M2300 viability from intra- and extracellular fractions of cultured ME180 cells using a gentamicin protection assay as described in the Methods. To quantify intracellular titers, the M. genitalium inoculum was incubated for $3 \mathrm{~h}$ to allow attachment to and entry of host cells (See Figure 1) followed by removal of the inoculum and replacement of fresh culture medium containing a bactericidal concentration of gentamicin $(200 \mathrm{ug} / \mathrm{mL})$. Fifteen minutes following inoculation with either G37 or M2300 (Figure 3), no viable M. genitalium were detected in the cells (data not shown). Using a color changing unit assay (CCU), high titers of viable intracellular M. genitalium were detected at both 24 h (not shown) and 48 h PI (Figure 3). No M. genitalium viability was detected in supernatants containing gentamicin at either point indicating that the observed titers were due exclusively to intracellular mycoplasmas that were protected from gentamicin exposure. Extracellular $M$. genitalium titers, representing organisms that had escaped from infected cells, were quantified from separate wells using supernatants of infected cells. Extracellular titers from culture supernatants (dashed line) were significantly less than intracellular titers $(p<0.05)$ at the tested time points ( $48 \mathrm{~h}$ shown in Figure 3 ). These data indicated that, after M. genitalium entry of the cell, more organisms remained inside the cell than egressed to the culture supernatant. Intracellular localization of M. genitalium in vaginal and cervical ECs also was consistent with electron microscopic analyses (Figure 1 and 2).

\section{M. genitalium elicited pro-inflammatory cytokines from genital epithelial cells}

Following demonstration of intracellular localization within reproductive tract ECs, we evaluated the host cytokine response from 3 human vaginal (V11I, V12I, and V19I) and 2 cervical EC lines (sA2EN and 3ECI) [16]. Of the tested time points, peak cytokine values were obtained $48 \mathrm{~h}$ PI and are presented in Table 1. Vaginal ECs exposed to viable M. genitalium G37 or M2300 (MOI 10) responded with significant secretion of interleukin-6 (IL6), IL-8 and GM-CSF ( $p<0.05$ vs. PBS control). Following M. genitalium exposure, ectocervical ECs secreted significant levels of IL-6 and IL-8 ( $\mathrm{p}<0.05$ vs. PBS control). Endocervical ECs responded to $M$. genitalium with the most number of secreted cytokines that included IL-6, IL8, G-CSF, GM-CSF and MCP-1 ( $p<0.05$ vs. PBS control). Using IL-8 secretion at $48 \mathrm{~h}$ PI as a comparator for all cell types, endocervical ECs were more responsive than vagi-

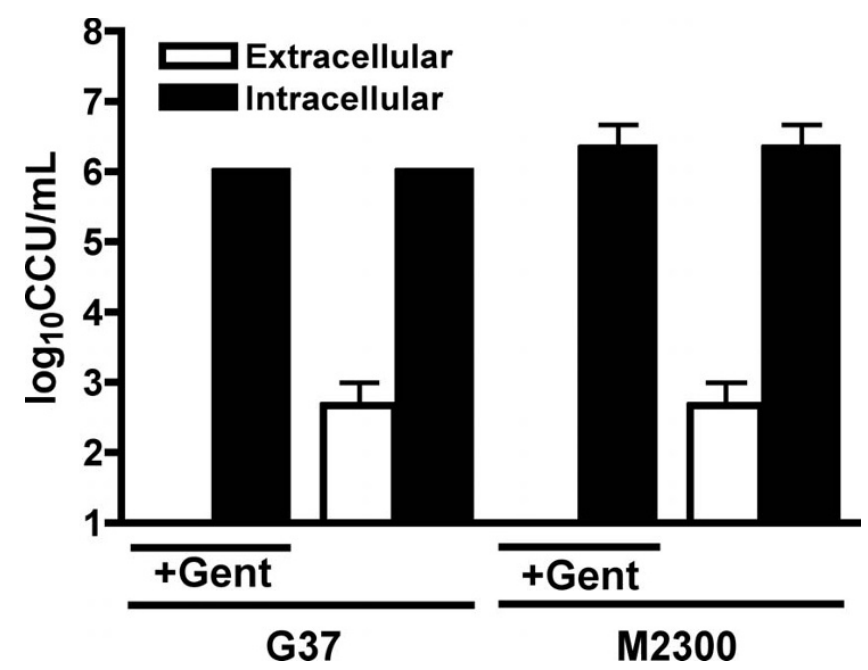

Figure 3

Intra- and extracellular localization of $M$. genitalium in ME- I 80 cervical epithelial cells. Cervical ECs (ME180) were inoculated with log-phase cultures of $M$. genitalium strain G37 (A) or M2300 (B) to determine whether M. genitalium can invade human reproductive tract ECs. To quantify intracellular M. genitalium loads (solid bar), the inoculum was removed following $3 \mathrm{~h}$ incubation for attachment and entry and replaced with medium containing gentamicin (200 ug/ $\mathrm{mL}$ ). The ability for $M$. genitalium to escape infected ECs (open bar) was quantified from culture supernatants in separate wells processed the same way except, following the $3 \mathrm{~h}$ incubation, the inoculum was removed and extracellular $M$. genitalium organisms were killed with gentamicin ( $2 \mathrm{~h}$ exposure). Infected cells then were washed thoroughly and received fresh medium without gentamicin allowing escaping M. genitalium to survive. Cell fractions or culture supernatants were collected at $48 \mathrm{~h}$ following removal of the inoculum for quantification of bacterial loads using a color changing unit (CCU) assay. In every case, significant differences between intracellular and extracellular $M$. genitalium titers were observed ( $p<0.05$; Student's t-test). Parallel studies were performed that employed $400 \mathrm{ug} / \mathrm{mL}$ gentamicin with similar results (data not shown).

nal or ectocervical cells when the fold increase of cytokine secretion by infected cells was calculated and compared to cells that received only PBS (ANOVA; $\mathrm{p}<0.01$, data not shown). A similar pattern of cytokine elaboration was observed following inoculation of M. genitalium at a MOI of 1 (data not shown). Cytokines that were not significantly induced by M. genitalium G37 or M2300 in any genital EC type included IL1-b, IL-2, IL-4, IL-5, IL-7, IL-9, IL10, IL-12(p40), IL-12(p70), IL-13，IL-15, IL-17, MIP1-a, MIP1-b, Basic FGF, Eotaxin, IP-10, PDGF-BB and VEG-F. The pattern of cytokines elaborated from cervical ECs was consistent with monocyte and macrophage recruitment and thus we next evaluated the responses of primary human MDM to M. genitalium exposure and determined 
Table I: Cytokine elaboration from human genital epithelial cells following M. genitalium $\mathbf{G} 37$ exposure ${ }^{a}$.

\begin{tabular}{|c|c|c|c|c|c|c|}
\hline & \multicolumn{2}{|c|}{$\begin{array}{c}\text { Vaginal } \\
\text { (VI9I, VI2I, VIII) }\end{array}$} & \multicolumn{2}{|c|}{$\begin{array}{l}\text { Ectocervical } \\
\text { (3ECl) }\end{array}$} & \multicolumn{2}{|c|}{$\begin{array}{l}\text { Endocervical } \\
\text { (sA2EN) }\end{array}$} \\
\hline & MOI IO & PBS & MOI IO & PBS & MOI IO & PBS \\
\hline IL-6 & $127 \pm 13 . I^{*}$ & $69 \pm 1.7$ & $63.7 \pm 1.8^{*}$ & $21.3 \pm 2.4$ & $348 \pm 13^{*}$ & $196 \pm 15$ \\
\hline IL-8 & $1458 \pm 117^{*}$ & $785 \pm 11.3$ & $3304 \pm 300 *$ & $722 \pm 98$ & $5 e 7 \pm 1347^{*}$ & $6 \mathrm{e} 4 \pm 367$ \\
\hline G-CSF & $261 \pm 46$ & $227 \pm 37$ & $548 \pm 143$ & $779 \pm 122$ & $155 \pm 6.2^{*}$ & $93 \pm 21$ \\
\hline GM-CSF & $24 \pm 1.8^{*}$ & $8 \pm 3.1$ & $16 \pm 2.6$ & $10 \pm 1.0$ & $160 \pm 9.4^{*}$ & $45 \pm 12$ \\
\hline MCP-I & $5.8 \pm 1.4$ & $7 \pm 2.1$ & $11.4 \pm 1.3$ & $10 \pm 3.1$ & $7.2 \pm 1.1^{*}$ & $0.46 \pm 0.02$ \\
\hline
\end{tabular}

aHuman vaginal $(n=3$ donors), ectocervical or endocervical ECs were inoculated with M. genitalium G37 (MOI 10). An equal volume of the PBS vehicle was added and processed in parallel as a control. Culture supernatants were collected $48 \mathrm{~h} \mathrm{PI}$ to quantify secreted cytokines. Values are expressed as the mean \pm SEM pg/mL from triplicate wells. Cytokine elaboration pattern and magnitudes induced following exposure to strain M2300 were not significantly different than G37. PBS values are presented to indicate basal cytokine elaboration from each cell type. ND, not detected. *, $p<0.05$ vs. PBS control using Student's t-test.

whether these cells were capable of M. genitalium phagocytosis and killing.

\section{Phagocytosis of M. genitalium by human monocyte- derived macrophages}

To determine the susceptibility of M. genitalium to macrophage phagocytosis, human MDM were exposed to logphase M. genitalium strains G37 or M2300 (MOI 100) and processed at selected time points for TEM. Within $5 \mathrm{~min}$ of inoculation, M. genitalium appeared dark staining with a dense content of ribosomes and no signs of membrane degeneration (Figure 4A). As early as $30 \mathrm{~min}$ PI, M. genitalium uptake by MDM was observed that was associated with morphologic changes of the bacterium including a loss of ribosome density and a hollow appearance (Figure 4B) consistent with a loss of viability. Internalized M. genitalium were prevalent and localized to membrane-bound phagolysosomes. Similar morphological changes were observed 2 h PI (data not shown). By 6 h PI, the macrophages appeared to have many phagocytic vesicles but no intracellular organisms could be located (Figure 4C). Viability of $M$. genitalium following macrophage exposure was evaluated by seeding infected MDM ( $6 \mathrm{~h} \mathrm{PI}$ ) into Friis FB medium at $37^{\circ} \mathrm{C}$. These cultures were observed for $M$. genitalium outgrowth by a $\mathrm{pH}$-mediated color change and microcolony formation. No growth was detected over a $14 \mathrm{~d}$ period from any of these cultures collectively indicating that M. genitalium was susceptible to rapid phagocytosis and killing.

\section{M. genitalium elicited pro-inflammatory cytokines from human monocyte-derived macrophages}

Because $M$. genitalium was phagocytosed rapidly by human MDM with no evidence of bacterial viability by 6 h PI, we sought to determine whether M. genitalium exposure to human MDM would elicit acute-phase cytokine responses. Viable M. genitalium G37 and M2300 inoculated at MOI 10 or MOI 1 elicited significant cytokine elaboration from macrophage cultures measured from supernatants collected $6 \mathrm{~h}$ PI (G37 [MOI 10] results presented in Table 2). No significant differences were observed between G37 and M2300 (data not shown). The profile of induced cytokine responses from human macrophages was composed predominately of early proinflammatory markers including significant secretion of IL-1 $\beta$, TNF- $\alpha$, IL-6, IL-8, G-CSF, IFN- $\gamma$, MCP-1, MIP-1 $\alpha$, MIP-1 $\beta$ and RANTES ( $\mathrm{p}<0.05$; Table 2$)$. These findings were consistent with results from 2 additional blood donors (data not shown). Following UV inactivation, $M$. genitalium elicited a similar profile and magnitude of cytokine secretion (Table 2) indicating that the immunostimulatory capacity was not dependent upon bacterial viability. Immune markers that were not induced by $M$. genitalium in human MDM included IL-2, IL-4, IL-5, IL-7, IL-9, IL-12(p70), IL-13, IL-15, IL-17, Basic FGF, Eotaxin, IP-10, PDGF-BB and VEG-F. Considering IL-6, a representative acute-phase cytokine with a central role in innate responses to bacterial pathogens [32], heat denaturation or proteinase-K digestion of $M$. genitalium significantly reduced the inflammatory capacity from human macrophages (Figure 5) suggesting that a significant proportion of the inflammatory capacity was mediated by M. genitalium protein components.

\section{Discussion}

Considering that $M$. genitalium reproductive tract infections in humans [1,33] and non-human primates [34] are often persistent, it seems likely that $M$. genitalium employs some tactic(s) to elude the host response to establish infection. Consistent with this hypothesis, attachment to and invasion of vaginal and cervical ECs by M. genitalium strains G37 and M2300 was observed by a subset of organisms as early as $2 \mathrm{~h}$ PI (Figure 1) suggesting that intracellular localization could provide a survival niche. The intracellular M. genitalium organisms were found in intracellular vacuoles similar in appearance to those seen in cultured Vero [27], HeLa and EM42 cells [35]. Approximately $60 \%$ of $M$. genitalium-containing vacuoles were 

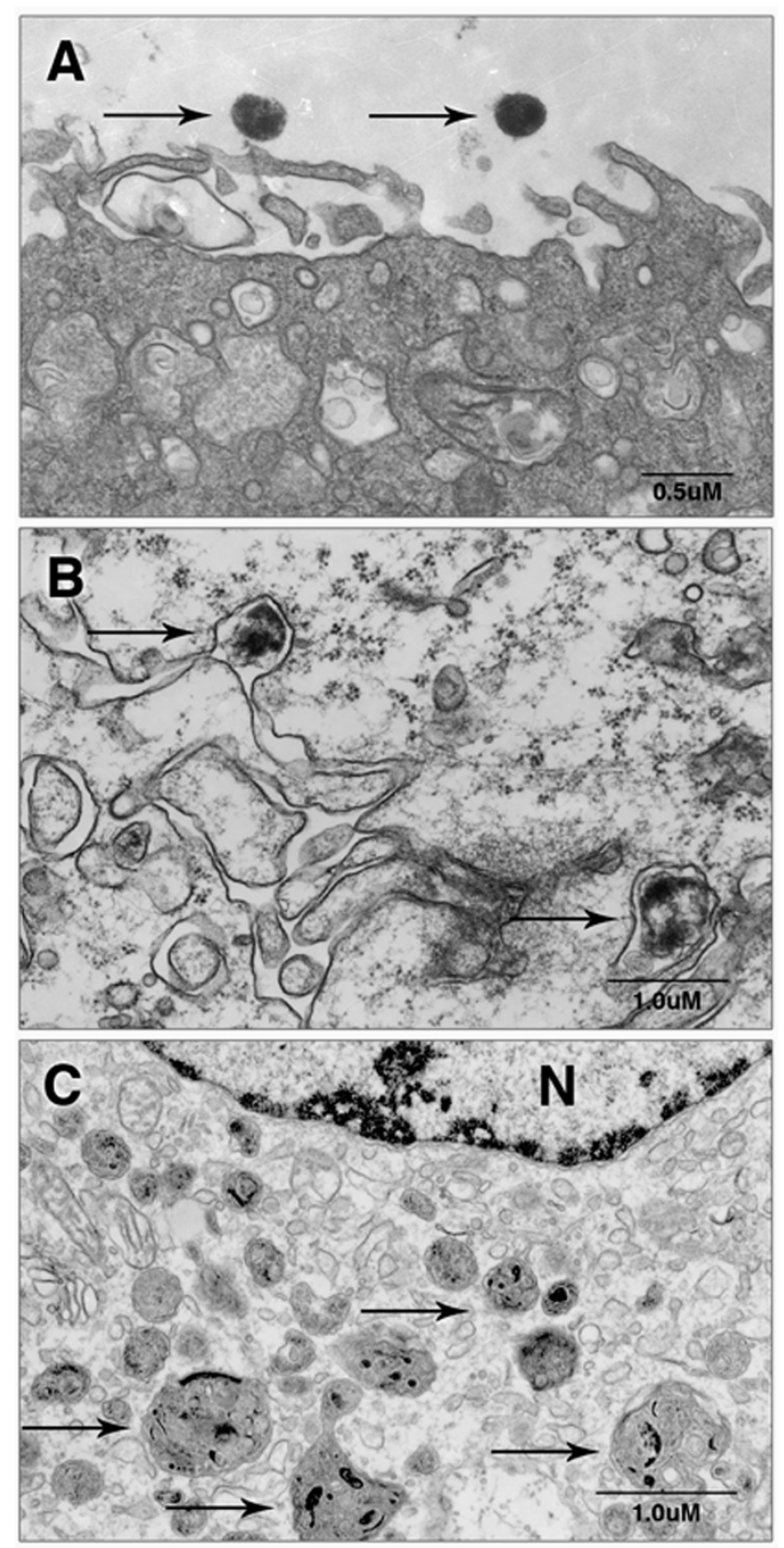

\section{Figure 4}

M. genitalium was phagocytosed rapidly by human monocyte-derived macrophages resulting in a loss of bacterial viability. Primary human MDM were inoculated with log-phase M. genitalium G37 or M2300 (MOI 100) and collected just after inoculation or $30 \mathrm{~min}$ or $6 \mathrm{~h} \mathrm{PI}$ and processed for TEM. Viable extracellular M. genitalium with dense intracellular ribosomes and an intact outer membrane were observed at the time of inoculation (A). Thirty minutes following inoculation, phagocytosis of $M$. genitalium was observed with localization to phagolysosomes (arrow) and morphological changes of the bacterium (B). By $6 \mathrm{~h} \mathrm{PI,} \mathrm{mac-}$ rophages contained many phagocytic vacuoles (arrows) and no intracellular mycoplasmas could be located (C). Micrographs depict $M$. genitalium strain $\mathrm{G} 37$ but similar findings were observed for strain $\mathrm{M} 2300$. $\mathrm{N}$ denotes nucleus. adjacent to the nucleus but also were distributed throughout the cytoplasm similar to a previous observation in cultured human endometrial cells [35]. Considering more than $20 \mathrm{~h}$ of microscope time and over 30 examined grids, it was concluded that more than $95 \%$ of cells showed attached M. genitalium organisms with roughly $50 \%$ of cells containing intracellular vacuoles with $M$. genitalium collected 0-48 h PI. Importantly, no M. genitalium organisms were ever observed free in the cytosol but were always bounded by a vacuolar membrane.

Our findings are the first report of intracellular localization in cultured human ECs from the vagina, ecto- and endocervix. These cell types are likely the first target cells following sexual transmission and therefore acute-phase interaction and host response are vital to understanding how M. genitalium establishes reproductive tract infection. The observation of $M$. genitalium invasion of vaginal and cervical ECs (Figure 1 and 2) is consistent with the clinical observation of heavy intracellular M. genitalium loads in PCR-positive vaginal specimens [30] and is substantiated by earlier reports of intracellular localization in cells of non-reproductive tract origin [27-30]. From our gentamicin invasion studies, $M$. genitalium was found both at intracellular sites and in extracellular fractions of infected cells. These outcomes were consistent with our electron microscopy studies as well. However, additional investigation will be required to address intracellular M. genitalium replication within host reproductive tract ECs as the experimental systems utilized for our studies did not facilitate reliable quantification of this outcome. Interestingly, it also was observed that, following intracellular localiza-

Table 2: Cytokine elaboration from human monocyte-derived macrophages following exposure to $M$. genitalium $\mathbf{G 3 7}^{a}$.

\begin{tabular}{cccc}
\hline \multicolumn{4}{c}{ Human MDM } \\
\hline & Viable & UV-Inactivated & PBS \\
\hline IL-I $\beta$ & $31 \pm 6 . I^{*}$ & $33 \pm 1.4^{*}$ & $0.7 \pm 0.04$ \\
IL-6 & $385 \pm 13.8^{*}$ & $439 \pm 4.0^{*}$ & $3.2 \pm 0.1$ \\
IL-8 & $5784 \pm 149^{*}$ & $5368 \pm 564^{*}$ & $116 \pm 7.8$ \\
G-CSF & $63.1 \pm 5.5^{*}$ & $72 \pm 2.4^{*}$ & $6.2 \pm 0.1$ \\
IFN- $\gamma$ & $270 \pm 24^{*}$ & $339 \pm 3.9^{*}$ & $9 \pm 3.6$ \\
MCP-I & $298 \pm 9.3^{*}$ & $318 \pm 8.3^{*}$ & $36 \pm 3.9$ \\
MIP-I $\alpha$ & $1056 \pm 16^{*}$ & $1068 \pm 4.0^{*}$ & $176 \pm 10.9$ \\
MIP-I $\beta$ & $2514 \pm 57^{*}$ & $2403 \pm 19^{*}$ & $810 \pm 47$ \\
RANTES & $66 \pm 1.5^{*}$ & $74 \pm 9.9^{*}$ & $11.4 \pm 0.4$ \\
TNF- $\alpha$ & $7456 \pm 334^{*}$ & $8616 \pm 697^{*}$ & $20 \pm 2.0$ \\
& & &
\end{tabular}

a Human MDM were inoculated with M. genitalium G37 (MOI I0) or an equal volume of the PBS vehicle as a control into triplicate wells. Culture supernatants were collected $6 \mathrm{~h} \mathrm{PI}$ to quantify secreted cytokines as described in the Methods. Values are expressed as the mean \pm SEM $\mathrm{pg} / \mathrm{mL}$ supernatant. PBS values are presented to indicate basal cytokine elaboration. Data collected following exposure to $M$. genitalium strain $\mathrm{M} 2300$ were similar in pattern and magnitude. ND, not detected. *, $\mathrm{p}<0.01$ using ANOVA. 


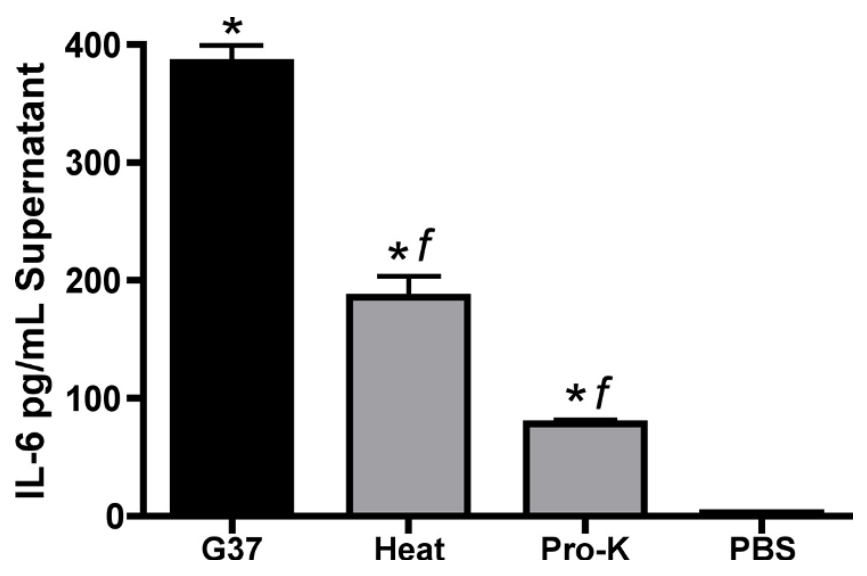

Figure 5

The $M$. genitalium-induced inflammatory cytokine secretion from human monocyte-derived macrophages was mediated predominately by proteins. Human MDM were exposed to viable M. genitalium G37 (MOI 10 ) or viable $M$. genitalium that had been denatured by heat or digested with proteinase-K (MOI I0). Cytokine secretion was quantified from culture supernatants collected $6 \mathrm{~h}$ following exposure as described in the Methods. Data shown are the mean \pm SEM of IL- 6 (a representative acutephase cytokine) induction from a typical experiment using $M$. genitalium strain $\mathrm{G} 37$ performed in triplicate wells compared to vehicle control (PBS) wells analyzed in parallel for each cell type. Data collected for each experiment using strain G37 or M2300 were similar in pattern and magnitude between 2 additional blood donors. *, $\mathrm{p}<0.01$ vs. PBS control using ANOVA. $f, p<0.01$ vs. viable $M$. genitalium G37.

tion by $M$. genitalium, a low level of egress from infected cells occurred (Figure 3) from 5-48 h PI suggesting that periodic egress from infected cells could result in cell to cell spread. Collectively, these results firmly indicate $M$. genitalium's capacity for invasion and prolonged intracellular survival that could provide the organism with a longterm survival niche in reproductive tract tissues.

From our studies of vaginal and cervical ECs, M. genitalium was observed at both intracellular and extracellular sites. However, it is not clear whether the invasive organisms are genetically different than those that were observed outside of the cells or whether some unknown factor facilitates entry of some organisms while excluding others. In addition, a well-defined tip structure [27,31] was rarely observed in our studies despite robust attachment to and invasion of the vaginal and cervical ECs (Figure 1 and 2) used in these studies. An area of increased electron density was observed within the $M$. genitalium organism (Figure 1C, F and 2) adjacent to the host cell surface presumably involved in attachment to the host cell. A similar structure has been described previously for M. genitalium strains grown attached to plastic cultureware [31].
These phenomena suggest that $M$. genitalium attachment to and invasion of reproductive tract ECs may not require a well-defined tip structure. In addition, attachment and invasion may involve cellular receptors that are localized to specific membrane sites that are better modeled using polarized 3-dimensional EC cultures. Indeed, the observed egress of $M$. genitalium from infected mucosal ECs likely would lead to infection of an adjacent cells in vivo rather than into the culture supernatant of traditional 2-dimensional cultures. This considered, a 3-dimensional multi-layer model of vaginal EC infection might better address how $M$. genitalium interacts with the host mucosa and establishes primary reproductive tract infection.

Because ECs likely serve as the first responders to STI, we investigated the acute-phase cytokine response to M. genitalium from human vaginal and cervical ECs. We found that M. genitalium elicited minimal innate responses from human vaginal ECs from 3 donors but ecto- and endocervical ECs were highly responsive and secreted cytokines consistent with recruitment of immune cells including IL8, G-CSF, GM-CSF and MCP-1 (Table 1). The increased responsiveness of endocervical ECs may have biological relevance, as the normally sterile upper tract tissues likely are more sensitive to microbial contamination than the lower genital tract. Paradoxically, it is in the upper tract tissues where inflammation due to microbial infection likely has the most severe consequences potentially leading to PID, salpingitis or reduced fertility [36]. Our studies were focused primarily on the lower genital tract but the heightened sensitivity of endocervical ECs provides rationale for testing cell types of the upper tract including endometrial [35] and fallopian ECs. All of the cell types used for cytokine analysis were immortalized by transduction of the human papilloma virus E6/E7 genes known to reduce the levels, but preserve the pattern of cytokine secretion relative to primary progenitor cells [16]. Therefore, we are confident that the observed cytokine inductions indicate the character of the responses but likely underestimate the actual levels of secretion.

Considering the profile of secreted cytokines by M. genitalium-infected reproductive ECs, we next investigated whether macrophages could play a role in the cellular immune response to $M$. genitalium. Following exposure to human MDM, phagocytosis of M. genitalium occurred rapidly (Figure 3 ) resulting in complete ablation of bacterial viability by 6 h PI. Importantly, several key pro-inflammatory cytokines were induced in response to $M$. genitalium exposure. IL- 6 secretion may be of particular importance considering that IL- 6 from vaginal secretions is positively correlated with HIV-1 burden [14] and known to up-regulate HIV-1 replication [15]. Indeed, the microbial burden of $M$. genitalium in clinically-obtained cervical specimens also is strongly associated with HIV-1 shedding 
[13]. Furthermore, macrophages are one of two major cellular reservoirs for latent HIV-1 infection and contribute to early-stage virus transmission and dissemination throughout the host (reviewed in [37]). To this end, we observed significant secretion of 4 potent chemokines responsible for granulocyte recruitment, MIP1-a, MIP1-b [38], MCP-1 and RANTES [39] (Table 2) indicating that macrophage exposure to $M$. genitalium in reproductive tissues likely would result in significant inflammation consistent with enhanced HIV-1 replication. Our findings suggest that both infected genital ECs and recruited immune cells are responsible for secretion of IL- 6 and other cytokines that may contribute to HIV-1 pathogenesis but continued research is necessary to dissect the cellular dynamics of HIV-1 and M. genitalium co-infections.

In our studies, the macrophage-stimulatory capacity of $M$. genitalium was not dependent upon bacterial viability. This outcome likely is due to the highly sensitive nature of macrophages. However, both heat denaturation and proteinase- $\mathrm{K}$ digestion significantly reduced the cytokine response (Figure 5) suggesting that a large proportion of M. genitalium's inflammatory capacity is indeed mediated by protein components. In addition, other findings from our group showed that $M$. genitalium and the antigenic MG309-encoded protein activate TLR2/ 6 to induce proinflammatory cytokine secretion from human MDM and reproductive tract ECs [22]. Collectively, these results indicated that macrophages are highly sensitive to M. genitalium exposure and highlight the putative pressure to evade the cellular immune responses.

Establishment of primary infection and persistence by $M$. genitalium in host tissues is not well understood. Our findings suggest that a subset of M. genitalium organisms rapidly invade host ECs thereby exploiting an intracellular survival niche to evade the potent and effective cellular host immune responses. Studies that address directly whether reproductive ECs provide protection from macrophage phagocytosis are currently underway and will be essential to understand this mechanism of immune evasion. Importantly, M. genitalium infection resulted in acute-phase inflammatory cytokine responses from vaginal and cervical ECs. Therefore, it is possible that persistent infection of female reproductive tract tissues may indeed result in inflammatory outcomes that could affect reproductive health but continued research is necessary to fully elucidate the mechanisms of $M$. genitalium-induced urogenital disease in women.

\section{Conclusion}

Human vaginal, ecto- and endocervical ECs were susceptible to M. genitalium G37 and M2300 infection resulting in rapid intracellular localization of a subset of organisms and significant secretion of pro-inflammatory cytokines.
The pattern of cytokine secretion was consistent with recruitment and stimulation of monocytes and macrophages at the vaginal and cervical mucosa. Following exposure to human monocyte-derived macrophages, $M$. genitalium was killed rapidly and elicited a potent proinflammatory response including secretion of cytokines associated with enhanced HIV-1 replication. These are the first data showing that cultured human vaginal and cervical ECs are susceptible and immunologically responsive to M. genitalium infection likely inducing cellular immune responses to infected tissues. Continued investigation of whether intracellular localization in reproductive tract ECs provides protection from the cellular immune response is warranted but rapid invasion of vaginal ECs, combined with the low immunological response, provides evidence for how $M$. genitalium might efficiently establish reproductive tract infection.

\section{Authors' contributions}

CLM carried out the intracellular dynamic studies, cytokine quantification assays, electron microscopy and drafted the manuscript. VLP provided assistance and direction in the study design and sample processing for electron microscopy. RBP participated in the study design, directed the overall research and helped draft the manuscript. All authors read and approved the final manuscript.

\section{Acknowledgements}

The authors thank Dr. Tonyia Eaves-Pyles and Michelle Kirtley from the UTMB Department of Microbiology and Immunology for their assistance with macrophage isolation. We also thank Violet Han and Julie Wen for their assistance in sample preparation for electron microscopy. We are grateful to Nicole Arrigo for critical reading of the manuscript. This work was supported by the Gulf South Sexually Transmitted Infection/Topical Microbicide Cooperative Research Center grant NIH-NIAID; UI9 Al061972.

\section{References}

I. Hjorth SV, Bjornelius E, Lidbrink P, Falk L, Dohn B, Berthelsen L, Ma L, Martin DH, Jensen JS: Sequence-based typing of Mycoplasma genitalium reveals sexual transmission. J Clin Microbiol 2006, 44(6):2078-2083.

2. Manhart LE, Holmes KK, Hughes JP, Houston LS, Totten PA: Mycoplasma genitalium among young adults in the United States: an emerging sexually transmitted infection. Am J Public Health 2007, 97(6): III8-II25.

3. Martin DH: Nongonococcal Urethritis: New Views through the Prism of Modern Molecular Microbiology. Curr Infect Dis Rep 2008, I 0(2): I 28-I32.

4. Haggerty CL: Evidence for a role of Mycoplasma genitalium in pelvic inflammatory disease. Curr Opin Infect Dis 2008, 2 I (I):65-69.

5. Falk L, Fredlund $H$, Jensen JS: Signs and symptoms of urethritis and cervicitis among women with or without Mycoplasma genitalium or Chlamydia trachomatis infection. Sex Transm Infect 2005, 8 I ( I ):73-78.

6. Manhart LE, Critchlow CW, Holmes KK, Dutro SM, Eschenbach DA Stevens CE, Totten PA: Mucopurulent cervicitis and Mycoplasma genitalium. J Infect Dis 2003, I 87(4):650-657.

7. Pepin J, Labbe AC, Khonde N, Deslandes S, Alary M, Dzokoto A, Asamoah-Adu C, Meda H, Frost E: Mycoplasma genitalium: an 
organism commonly associated with cervicitis among west African sex workers. Sex Transm Infect 2005, 8 I (I):67-72.

8. Uno M, Deguchi T, Komeda H, Hayasaki M, lida M, Nagatani M, Kawada $Y$ : Mycoplasma genitalium in the cervices of Japanese women. Sex Transm Dis 1997, 24(5):284-286.

9. Korte JE, Baseman JB, Cagle MP, Herrera C, Piper JM, Holden AE, Perdue ST, Champion JD, Shain RN: Cervicitis and genitourinary symptoms in women culture positive for Mycoplasma genitalium. Am J Reprod Immunol 2006, 55(4):265-275.

10. Cohen CR, Mugo NR, Astete SG, Odondo R, Manhart LE, Kiehlbauch JA, Stamm WE, Waiyaki PG, Totten PA: Detection of Mycoplasma genitalium in women with laparoscopically diagnosed acute salpingitis. Sex Transm Infect 2005, 8 I (6):463-466.

II. Clausen HF, Fedder J, Drasbek M, Nielsen PK, Toft B, Ingerslev HJ, Birkelund S, Christiansen G: Serological investigation of Mycoplasma genitalium in infertile women. Hum Reprod 200I, 16(9):1866-1874.

12. Svenstrup HF, Fedder J, Kristoffersen SE, Trolle B, Birkelund S, Christiansen G: Mycoplasma genitalium, Chlamydia trachomatis, and tubal factor infertility-a prospective study. Fertil Steril 2008, 90(3):5।3-520.

13. Manhart LE, Mostad SB, Baeten JM, Astete SG, Mandaliya K, Totten PA: High Mycoplasma genitalium organism burden is associated with shedding of HIV-I DNA from the cervix. J Infect Dis 2008, I 97(5):733-736.

14. Al-Harthi L, Kovacs A, Coombs RW, Reichelderfer PS, Wright D, Cohen MH, Cohn J, Cu-Uvin S, Watts H, Lewis S, et al.: A menstrual cycle pattern for cytokine levels exists in HIV-positive women: implication for HIV vaginal and plasma shedding. Aids 200I, I 5( I 2): I535-1543.

15. Copeland KF: Modulation of HIV-I transcription by cytokines and chemokines. Mini Rev Med Chem 2005, 5(I 2): I093-I I I I.

16. Herbst-Kralovetz MM, Quayle AJ, Ficarra M, Greene S, Rose WA 2nd, Chesson R, Spagnuolo RA, Pyles RB: Quantification and comparison of toll-like receptor expression and responsiveness in primary and immortalized human female lower genital tract epithelia. Am J Reprod Immunol 2008, 59(3):2I 2-224.

17. Fichorova RN, Cronin AO, Lien E, Anderson DJ, Ingalls RR: Response to Neisseria gonorrhoeae by cervicovaginal epithelial cells occurs in the absence of toll-like receptor 4mediated signaling. J Immunol 2002, I68(5):2424-2432.

18. Givan AL, White HD, Stern JE, Colby E, Gosselin EJ, Guyre PM, Wir CR: Flow cytometric analysis of leukocytes in the human female reproductive tract: comparison of fallopian tube, uterus, cervix, and vagina. Am J Reprod Immunol 1997 38(5):350-359.

19. Kaufmann SHE, Medzhitov R, Gordon S: The Biology of Macrophages. In The Innate Immune Response to Infection ASM Press, Washington, DC; 2004:7I-72.

20. Wu Y, Qiu H, Zeng Y, You X, Deng Z, Yu M, Zhu C: Mycoplasma genitalium lipoproteins induce human monocytic cell expression of proinflammatory cytokines and apoptosis by activating nuclear factor kappaB. Mediators Inflamm 2008: 195427.

21. You X, Wu Y, Zeng Y, Deng Z, Qiu H, Yu M: Mycoplasma genitalium-derived lipid-associated membrane proteins induce activation of MAPKs, NF-kappaB and AP-I in THP-I cells. FEMS Immunol Med Microbiol 2008, 52(2):228-236.

22. McGowin CL, Ma L, Martin DH, Pyles RB: Mycoplasma genitalium-encoded MG309 activates NF-\{kappa\}B via Toll-like receptor 2/6 to elicit pro-inflammatory cytokine secretion from human genital epithelial cells. Infect Immun 2008, 77(3): $1175-1181$.

23. Quayle A): The innate and early immune response to pathogen challenge in the female genital tract and the pivotal role of epithelial cells. J Reprod Immunol 2002, 57(I-2):6 I-79.

24. Jensen JS, Hansen HT, Lind K: Isolation of Mycoplasma genitalium strains from the male urethra. J Clin Microbiol 1996, 34(2):286-29l.

25. Soler-Rodriguez AM, Zhang H, Lichenstein HS, Qureshi N, Niesel DW, Crowe SE, Peterson JW, Klimpel GR: Neutrophil activation by bacterial lipoprotein versus lipopolysaccharide: differential requirements for serum and CDI4. I Immunol 2000, 164(5):2674-2683.

26. Elsinghorst EA: Measurement of invasion by gentamicin resistance. Methods Enzymol 1994, 236:405-420.
27. Jensen JS, Blom J, Lind K: Intracellular location of Mycoplasma genitalium in cultured Vero cells as demonstrated by electron microscopy. Int J Exp Pathol 1994, 75(2):91-98.

28. Mernaugh GR, Dallo SF, Holt SC, Baseman JB: Properties of adhering and nonadhering populations of Mycoplasma genitalium. Clin Infect Dis 1993, I 7(SuppI I):S69-78.

29. Baseman JB, Lange M, Criscimagna NL, Giron JA, Thomas CA: Interplay between mycoplasmas and host target cells. Microb Pathog 1995, 19(2): 105-II6.

30. Blaylock MW, Musatovova O, Baseman JG, Baseman JB: Determination of infectious load of Mycoplasma genitalium in clinical samples of human vaginal cells. J Clin Microbiol 2004, 42(2):746-752.

31. Pich OQ, Burgos R, Ferrer-Navarro M, Querol E, Pinol J: Role of Mycoplasma genitalium MG218 and MG317 cytoskeletal proteins in terminal organelle organization, gliding motility and cytadherence. Microbiology 2008, I54(Pt 10):3।88-3198.

32. Jones SA: Directing transition from innate to acquired immunity: defining a role for IL-6. J Immunol 2005, I75(6):3463-3468.

33. Cohen CR, Nosek M, Meier A, Astete SG, Iverson-Cabral S, Mugo NR, Totten PA: Mycoplasma genitalium infection and persistence in a cohort of female sex workers in Nairobi, Kenya. Sex Transm Dis 2007, 34(5):274-279.

34. Taylor-Robinson D: The Harrison Lecture. The history and role of Mycoplasma genitalium in sexually transmitted diseases. Genitourin Med 1995, 7I(I): I-8.

35. Ueno PM, Timenetsky J, Centonze VE, Wewer JJ, Cagle M, Stein MA, Krishnan M, Baseman JB: Interaction of Mycoplasma genitalium with host cells: evidence for nuclear localization. Microbiology 2008, I54(Pt I0):3033-304I.

36. Haggerty CL, Ness RB: Epidemiology, pathogenesis and treatment of pelvic inflammatory disease. Expert Rev Anti Infect Ther 2006, 4(2):235-247.

37. Carter CA, Ehrlich LS: Cell biology of HIV-I infection of macrophages. Annu Rev Microbiol 2008, 62:425-443.

38. Sherry B, Tekamp-Olson P, Gallegos C, Bauer D, Davatelis G, Wolpe SD, Masiarz F, Coit D, Cerami A: Resolution of the two components of macrophage inflammatory protein $I$, and cloning and characterization of one of those components, macrophage inflammatory protein I beta. J Exp Med 1988, I 68(6):2251-2259.

39. Navratilova Z: Polymorphisms in CCL2\&CCL5 chemokines/ chemokine receptors genes and their association with diseases. Biomed Pap Med Fac Univ Palacky Olomouc Czech Repub 2006 , I50(2): $|9|-204$

Publish with Biomed Central and every scientist can read your work free of charge

"BioMed Central will be the most significant development for disseminating the results of biomedical research in our lifetime. "

Sir Paul Nurse, Cancer Research UK

Your research papers will be:

- available free of charge to the entire biomedical community

- peer reviewed and published immediately upon acceptance

- cited in PubMed and archived on PubMed Central

- yours - you keep the copyright
BioMedcentral 